\title{
Primary pulmonary artery sarcoma versus pulmonary thromboembolism: a multimodal imaging comparison
}

\author{
Ellen Kronzer ${ }^{1} \cdot$ Steven I. Robinson ${ }^{2} \cdot$ Douglas A. Collins $^{3} \cdot$ Robert D. McBane II ${ }^{4,5}$ (i)
}

Accepted: 26 April 2021 / Published online: 7 May 2021

(c) The Author(s), under exclusive licence to Springer Science+Business Media, LLC, part of Springer Nature 2021

\begin{abstract}
Primary pulmonary artery sarcoma (PPAS) is a rare malignancy that is commonly mistaken for pulmonary embolism due to similarities in clinical presentation and radiographic findings. Distinct radiographic findings to help differentiate between the two diseases are highlighted in the case presented. (1) Several nuances in various imaging modalities have been identified to help distinguish pulmonary artery sarcoma from pulmonary thromboembolic disease. (2) The wall eclipsing sign is considered pathognomonic for pulmonary artery sarcoma. (3) Positron emission tomography/computed tomography may help reduce time between diagnosis and treatment, which may ultimately prolong survival. (4) Providers should be well versed on the subtle differences on imaging to prevent future delays in diagnosis and treatment.
\end{abstract}

Keywords Emboli $\cdot$ Sarcoma $\cdot$ Malignancy $\cdot$ Imaging

\section{Highlights}

- Pulmonary artery sarcoma is a rare malignancy which can be confused radiographically with pulmonary embolism.

- Several clues on imaging can help distinguish these two entities including the "wall eclipsing sign", lobulated bulging margins, gadolineum enhancement during MRI imaging, and FDG uptake during PET imaging.

- Prompt and accurate diagnosis may improve survival for resectable tumors.

Robert D. McBane II

mcbane.robert@mayo.edu

1 Department of Internal Medicine, Mayo Clinic, Rochester, MN 55905, USA

2 Medical Oncology, Mayo Clinic, Rochester, MN 55905, USA

3 Department of Radiology, Mayo Clinic, Rochester, MN 55905, USA

4 Gonda Vascular Center, Mayo Clinic, 200 First Street SW, Rochester, MN 55905, USA

5 Department of Cardiovascular Disease, Mayo Clinic, Rochester, MN 55905, USA

\section{Case report}

A 63-year-old female Jehovah's Witness was evaluated for a persistent dry cough, dyspnea, and pleuritic chest pain. Influenza, respiratory syncytial virus, and COVID-19 testing were negative. Chest $\mathrm{x}$-ray was concerning for pneumonia; however, symptoms were unresponsive to antibiotics. Chest computed tomography (CT) with contrast revealed a large filling defect within the right lobar, upper and middle segmental pulmonary arteries with central calcification. She was initiated on apixaban for suspected pulmonary embolism, but her symptoms persisted. Repeat CT angiography 6 weeks later revealed proximal extension of the filling defect despite adherence to medical therapy (Figs. 1 and 2), prompting referral to the Mayo Clinic Gonda Vascular Center.

Upon arrival to Mayo Clinic, patient was transitioned to enoxaparin. Further evaluation included a complete thrombophilia panel which was negative for congenital or acquired thrombotic propensity, including a negative D-dimer. Lower extremity venous ultrasound was negative for deep vein thrombosis. Positron emission tomography (PET) revealed an F-18 fluorodeoxyglucose (FDG) avid right hilar mass with a satellite lesion adjacent to the pleura (Fig. 3). Cardiac magnetic resonance imaging (MRI) revealed a large mass within the right pulmonary artery extending into the right lobar segmental arteries with central gadolinium 


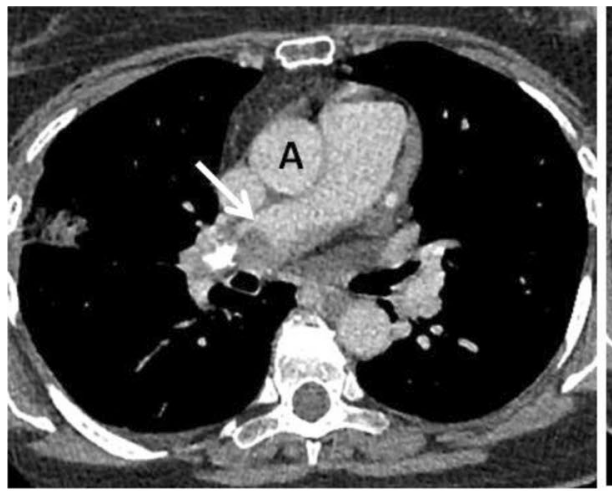

May 2020

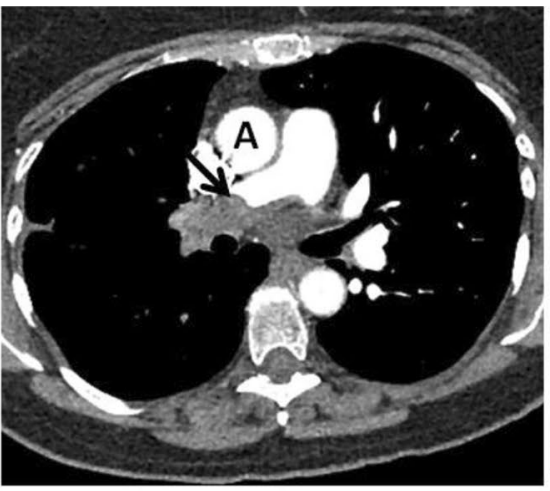

July 2020

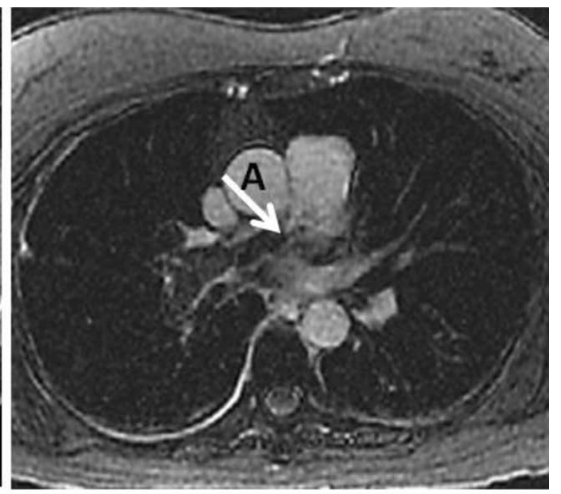

August 2020
Fig. 1 Pulmonary artery filling defect progression. Over the 3 month interval from May through August, 2020, there was clear progression of the right pulmonary artery filling defect. May (Left Panel) and July 2020 (Center Panel) imaging modalities were contrast enhanced chest computed tomography (CT). The August 2020 modality (Right Panel) was MRI with gadolinium. The interval growth of the filling defect is best appreciated by comparison of the proximal edge (arrow) to the adjacent ascending aorta (A)

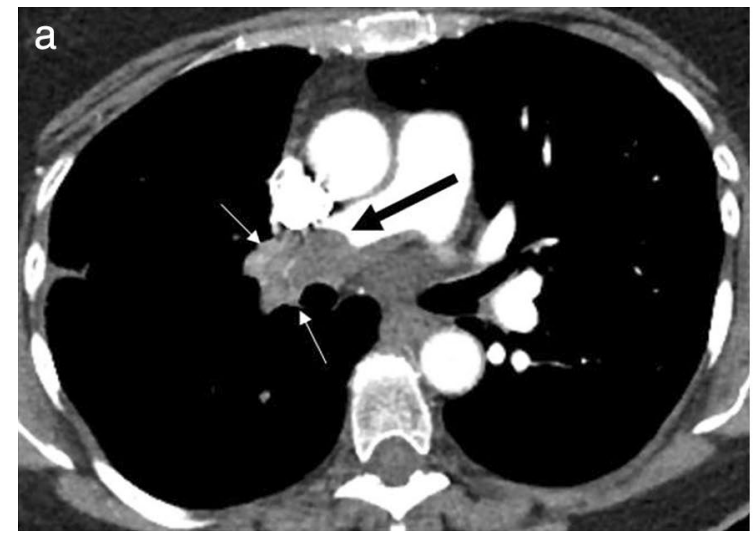

Fig. 2 "Wall Eclipsing Sign" by CT Angiography. These images demonstrate the wall eclipsing sign (Panel a) including involvement of the right pulmonary artery with near complete luminal occlusion (thick black arrow) and propagation toward the right ventricular out-

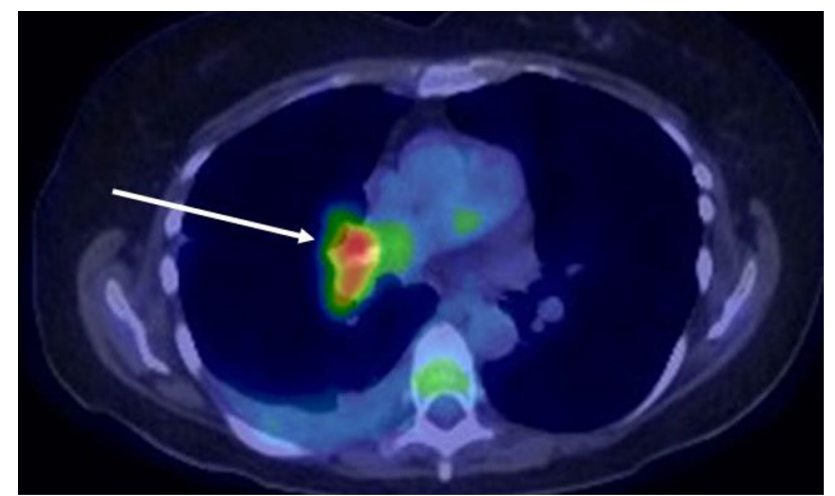

Fig. 3 PET CT Imaging with F-18 FDG. F-18 Fluorodeoxyglucose (FDG) avid soft tissue mass (arrow) involving the right pulmonary artery extends into right lobar arteries with central calcification compatible with malignancy

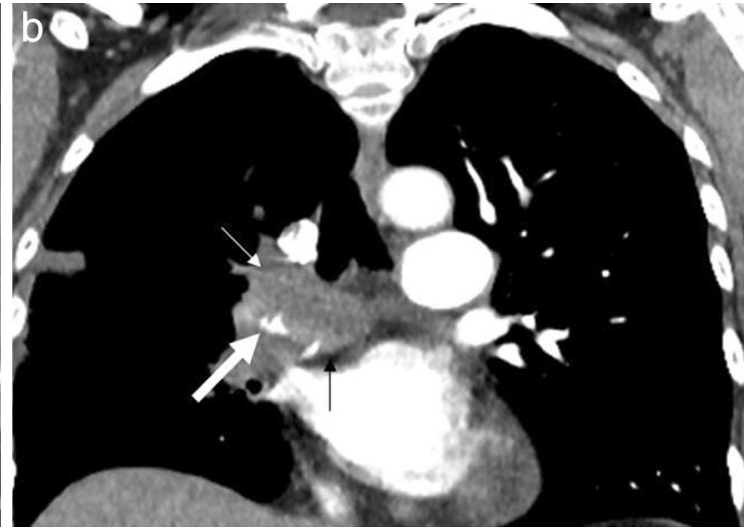

flow tract. The "eclipsing" of the pulmonary artery with lesion extension beyond the arterial wall boundary (thin white arrows) is demonstrated in the coronal imaging (Panel b). The thick arrow in Panel b demonstrates intra-mass calcification

enhancement and interval enlargement of a paratracheal lymph node (Fig. 4). Endobronchial ultrasound needle biopsy confirmed high grade pleomorphic primary pulmonary artery sarcoma.

When multidisciplinary evaluation deemed primary surgical resection to be prohibitive due to patient refusal to receive blood product support, chemotherapy with doxorubicin, ifosfamide with mesna growth factor support was initiated. PET/CT at 3-month follow-up showed partial metabolic response with no evidence of new FDG avid metastatic disease. Cardiac MRI demonstrated decrease in tumor size. 


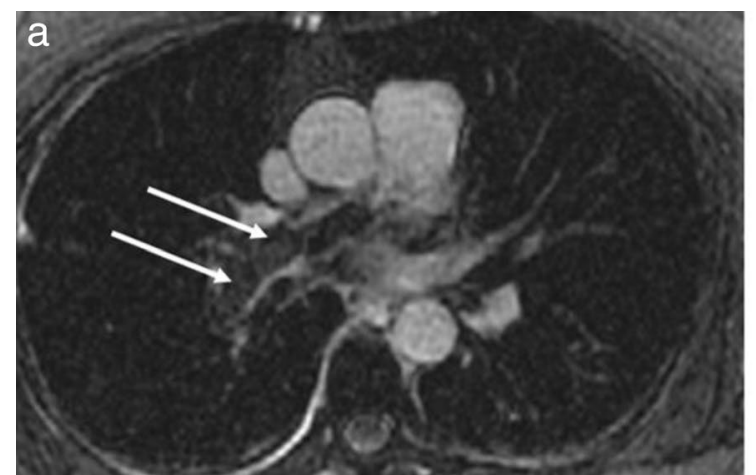

Fig. 4 Gadolinium Enhanced 4D Cardiac MRI. Central gadolinium enhancement of the right pulmonary artery mass is most consistent with tumor thrombus (Panel a, arrows) and adjacent bland thrombus.

\section{Discussion}

Primary pulmonary artery sarcoma (PPAS) is a rare and often fatal malignancy with an estimated global incidence of $0.001-0.03 \%$ [1]. Age of presentation ranges from 13 to 86 years old, though peak incidence occurs between ages $45-55$ and a female predominance $(2: 1)[1,2]$. The prognosis of PPAS is poor with a survival period of 12-18 months from symptom onset and mean survival $<2$ months without surgical intervention [3]. Its poor prognosis is heavily influenced by its frequent delay in diagnosis due to its tendency to mimic chronic thromboembolic disease in both clinical and radiologic findings [2-5]. Nuanced clues on imaging studies can alert providers to the presence of PPAS and help distinguish this malignancy from pulmonary embolism. With these clues, early recognition may help expedite diagnosis and treatment (Table 1).

Pulmonary artery CT angiography has been found to be a useful tool in distinguishing between PPAS and pulmonary thromboembolic disease. While both can result in filling defects of the pulmonary arteries, PPAS can extend into the main pulmonary trunk and right ventricular outflow tract, tends to occupy the entire lumen with local aneurysmal dilatation, and may form characteristic acute angles with the vessel wall [1-6]. PPAS tumors also typically have lobulated, bulging margins proximally whereas pulmonary thromboemboli typically have straight, cup-shaped margins $[1,2,4-6]$. Furthermore, approximately $90 \%$ of PPAS lesions involve $\geq 2$ parts of pulmonary arteries; most commonly affecting the lobar pulmonary artery $(85 \%)$, followed thereafter by the right pulmonary artery $(71 \%)$, left pulmonary artery $(65 \%)$, and right ventricular outflow tract $(10 \%)$ [1].

The "wall eclipsing sign" on CT angiography may be helpful in distinguishing PPAS from thromboembolic disease [7]. This sign is defined as the presence of the following three findings: a low-density intraluminal mass of the

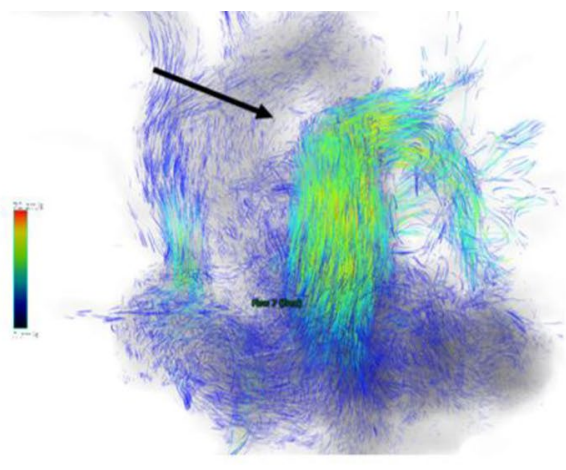

Slow flow in the area of the mass with 4D flow analysis (Panel b, arrow) demonstrates little to no blood flow present through the right pulmonary artery proximally

pulmonary trunk, left pulmonary artery, or right pulmonary artery with near complete occlusion; proximal protrusion of the mass toward the right ventricular outflow tract; and eclipsing of one or both walls of the involved artery before the lesion infiltrates beyond the artery. In one study, this sign was assessed in 12 patients with PPAS compared to 156 patients with chronic pulmonary thromboembolic (CPTE) disease with pulmonary hypertension and 426 patients with acute pulmonary embolism. The "wall eclipsing sign" was present in all PPAS patients, but none of the patients with either acute or chronic thromboembolic disease [7].

Gadolinium enhanced MRI may also be useful to differentiate between PPAS and pulmonary thromboembolic disease. Enhancement of a lesion on gadolinium-MRI is consistent with tumor [1]. PPAS tumors tend to display a heterogeneous delayed enhancement pattern with a gradual rise in time-signal intensity curves, as well as a higher T2 signal intensity compared to PTE $[1,2,4,5]$. MRI also provides valuable information regarding the pulmonary artery intima. A study done by Ming-Xi Liu et al. observed increased intimal thickening adjacent to PAS tumors with indistinct margins secondary to intimal invasion, a feature not identified with CPTE disease. While calcification may be present in thrombotic disease, it typically signifies a chronic process [1].

FDG PET/CT may be helpful to determine the presence of malignancy. Similar to evaluating a lesion for enhancement on gadolinium-MRI, FDG uptake on PET/CT can be used to differentiate between PPAS and PTE as FDG activity will be more intense in PPAS compared to thromboembolic disease [2, 3]. FDG PET/CT can also facilitate reducing the time between diagnosis and treatment when utilized as part of the diagnostic work-up [8]. Tueller et al. group described a series of patients in which FDG PET/CT was used to simultaneously diagnose PPAS and provide initial staging, ultimately accelerating the timeline for curative resection. 
Table 1 Subtle imaging differences identified on various imaging modalities to differentiate between pulmonary artery sarcoma and pulmonary thromboembolic disease

\begin{tabular}{|c|c|c|}
\hline & Pulmonary artery sarcoma & Pulmonary thromboembolism \\
\hline Pulmonary artery CTA & $\begin{array}{l}\text { Involves } \geq 2 \text { pulmonary arteries, most often } \\
\text { including the main pulmonary trunk } \\
\text { Local aneurysmal dilatation } \\
\text { Proximal lobulated, bulging margins } \\
\text { Wall eclipsing sign }\end{array}$ & $\begin{array}{l}\text { Involves the right and/or } \\
\text { left pulmonary arteries, or } \\
\text { saddle } \\
\text { Proximal straight, cup-shaped } \\
\text { margins } \\
\text { Absence of wall eclipsing } \\
\text { sign }\end{array}$ \\
\hline MRI & $\begin{array}{l}\text { Heterogeneous delayed central enhancement } \\
\text { High T2 signal intensity } \\
\text { Intimal wall thickening with discontinuity }\end{array}$ & $\begin{array}{l}\text { Little to no enhancement } \\
\text { Clear discrimination between } \\
\text { lesion and adjacent intima }\end{array}$ \\
\hline FDG PET/CT & Increased FDG uptake & Decreased FDG uptake \\
\hline
\end{tabular}

\section{Conclusion}

In conclusion, primary pulmonary artery sarcomas are often mistaken for pulmonary thromboembolic disease given their similarities in clinical presentation and radiologic findings; however, there are subtle imaging characteristics that can help differentiate the two entities. Recognizing these radiologic features may expedite patient diagnosis and subsequent treatment. Radical surgical resection is the mainstay treatment for PPAS and early diagnosis is key in prolonging survival. Of the chemotherapy trials that have been performed, few have shown any definite response. Perioperative chemotherapy may provide some survival benefit, but studies are limited. For those with inoperable PPAS as seen in our patient, palliative chemo- and radiotherapy should be considered [9].

Funding No funding to report.

\section{Declarations}

Conflict of interest The above authors have nothing to disclose.

\section{References}

1. Liu MX, Ma ZH, Jiang T, Guo XJ, Yu FF, Yang YH, Zhai ZG (2018) Differential diagnosis of pulmonary artery sarcoma and central chronic pulmonary thromboembolism using CT and MR images. Heart Lung Circ 27(7):819-827. https://doi.org/10.1016/j. hlc.2017.06.716
2. Moguillansky NI, Verma N, Shah P, Knapik J, Mohammed TL (2019) Pulmonary artery sarcoma: case report and review of the literature. Respir Med Case Rep 27:100857. https://doi.org/10. 1016/j.rmcr.2019.100857

3. Jeong N, Seol SH, Kim IH, Kim JY (2016) Pulmonary artery sarcoma-multimodality imaging. J Clin Imaging Sci 6:45. https:// doi.org/10.4103/2156-7514.192841

4. Djordjevic I, Pejcic T, Rancic M, Radovic M, Bosnjakovic P, Radjenovic-Petkovic T, Nastasijevic-Borovac D, Golubovic S, Dacic D (2009) Difficulties in establishing a timely diagnosis of pulmonary artery sarcoma misdiagnosed as chronic thrombo-embolic pulmonary disease: a case report. J Med Case Rep 3:64. https:// doi.org/10.1186/1752-1947-3-64

5. Bandyopadhyay D, Panchabhai TS, Bajaj NS, Patil PD, Bunte MC (2016) Primary pulmonary artery sarcoma: a close associate of pulmonary embolism-20-year observational analysis. J Thorac Dis 8(9):2592-2601. https://doi.org/10.21037/jtd.2016.08.89

6. Liu X, Hou J, Wang X, Chen Z (2017) An intimal sarcoma of pulmonary artery mimicking pulmonary embolism: a case report and literature review. Respirol Case Rep 5(5):e00248. https://doi. org/10.1002/rcr2.248

7. Gan HL, Zhang JQ, Huang XY, Yu W (2013) The wall eclipsing sign on pulmonary artery computed tomography angiography is pathognomonic for pulmonary artery sarcoma. PLoS ONE 8(12):e83200. https://doi.org/10.1371/journal.pone.0083200

8. Tueller C, Fischer BR, Minder S (2010) FDG-PET in diagnostic work-up of pulmonary artery sarcomas. Eur Respir J 35:444-446. https://doi.org/10.1183/09031936.00114708

9. Wong H, Gounaris I, McCormack A, Berman M, Davidson D, Horan G, Pepke-Zaba J, Jenkins D, Earl H, Hatcher H (2015) Presentation and management of pulmonary artery sarcoma. Clin Sarcoma Res 5:3. https://doi.org/10.1186/s13569-014-0019-2

Publisher's Note Springer Nature remains neutral with regard to jurisdictional claims in published maps and institutional affiliations. 